\title{
Applying the Paschalidis Tri-Anthropo-Type Model in the Kindergarten: A Case Study
}

\author{
G. D. Paschalidis \\ Researcher, Author, Greece \\ D. Avlogiari \\ Pre-School Teacher, Greece
}

\author{
K. Chiou \\ Coordinator of Psychological Rehabilitation \\ Centre, Greece
}

P. Karaviti

Secondary School Teacher, Theatre/Drama Educator, Greece

\begin{abstract}
This study examines the effect of the application of the Tri-Anthropo-Type Paschalidis Model on preschool education children. According to the Model, people have specific personality traits, defined by their brain function. As a result, they belong to one of three distinct personality Types ( $A, B$ and $C$ ), with certain physical and psychological characteristics, as well as certain cognitive and learning abilities. In the kindergarten class where the Model was applied, the teacher - researcher had encountered incidents of abusive behavior and lack of collaboration amongst children, which made her professional life stressful and rather unpleasant at times. There were also certain behavioral problems and developmental disorders, which needed to be dealt with. The action research provided us with evidence that the intervention assisted in the reduction of such behavioral problems, facilitated children's selfawareness and hetero-awareness and contributed to the creation of a cooperative and friendly learning environment. The children's acquisition of essential social skills was achieved effectively and rapidly. The intervention contributed to the children's positive change, namely self-respect and selfconfidence, by helping them accept their own individuality, rely on their strengths and minimize their weaknesses. The class teacher also benefited from the intervention, in that she could work in a happier environment.
\end{abstract}

\section{Introduction}

In the beginning of the school year 2013-14, in the teacher's kindergarten class, a number of children used to abuse each other verbally and/or physically and to find it hard to share and collaborate. In the past, when facing similar situations, the teacher used to approach each child's problematic behavior as unique. She soon realized, however, that this approach constituted a time and energy consuming process, which deprived other class children of her attention and care, and which wasn't always effective. When she came across the Tri-Anthropo-Type Paschalidis Model in the literature, she thought that her class could benefit from it [1]. According to the Model, every person belongs to only one of three distinct personality Types, A, B or C, differentiated mainly by brain function and plasticity [2]. The Paschalidis Model can be regarded as a development of Plato's division of people in the Republic. Type A personalities correspond to Plato's philosophers/kings, Type B to the guardians and Type $\mathrm{C}$ to the producers. The Paschalidis Model goes beyond Plato's theoretical concept of an ideal state. it constitutes a practical application liberating people from the limitations imposed by their personality Type. In an educational context, these three personality Types define three distinct learning profiles, as well as three distinct teaching approaches. When children do not face stressful situations, they exhibit the best qualities of their personality. Type A children are independent, they adapt easily to changes and share their belongings. Type B children are polite, patient, sociable and enjoy offering help and advices. And Type $\mathrm{C}$ children are concerned passionate and daring, have a strong will and a sense of justice. They do not give up easily.

Under stress children exhibit the worst qualities of their personality, which constitute each personality Types' weaknesses. Every personality Type (A, B or C) has a specific reaction to stress related stimuli. Specifically, when under stress, Type A children are prone to panic, irritability and 
hyperactivity, Type B children become anxious, worry about the future and procrastinate, and Type $\mathrm{C}$ children become stubborn, inflexible and aggressive [3].

According to the Model, the three personality Types, A, B and C, are classified according to certain physiological, neuroanatomical and neurochemical characteristics, which are genetically specified and inherited by one's biological parents [2]. The fundamental differentiating element, though, is a distinct Type -specific brain function. Recent neuroscience research on regions of the limbic system suggest that brain abnormalities in schizophrenia involve medial temporal lobe structures and neocortical temporal lobe regions, the amygdala gland plays a significant role in anxiety during early childhood and the hippocampus is connected with obsessive-compulsive type disorders [4] [5] [6]. According to the Paschalidis Model, as far as information processing and action are concerned, Type A individuals, whose entorhinal cortex gets overactive under stress, process information instantly and act immediately, Type B individuals, whose amygdala gland gets overactive under stress, process information cautiously and hesitate to act, and Type $\mathrm{C}$ individuals, whose hippocampus gets overactive under stress, process information obsessively and act persistently.

According to the Model, children of each personality Type show different signs of what is defined as ADHD. Specifically, Type A children show signs of hyperactivity, Type B children show signs of attention deficit disorder and Type $\mathrm{C}$ children suffer from Asperger disorder [3].

The Model introduces what it defines as the "dominant gene" factor, which also plays significant role in Type-specific characteristics. Despite the fact that there are only three personality Types, and therefore three distinct Types of idiosyncrasies (A Type characterized by action, B Type by nervousness and $\mathrm{C}$ Type by order, dogmatic), there are six sub-types depending on whether one has inherited their personality Type from their mother or their father. In the Model this is known as "dominant gene".

The dominant gene defines the way each person handles and manages their life and relations. People who have inherited their dominant gene from their father, they exhibit a male attitude as far as their behavior, actions and reactions are concerned, no matter the personality Type they belong to. They tend to be more tense and abrupt, extrovert and lacking diplomacy skills. People who have inherited their dominant gene from their mother, they exhibit a female attitude as far as their behavior, actions and reactions are concerned, no matter the personality
Type they belong to. They tend to express themselves in a softer and gentler manner, are analytical, introvert and accepting. When the dominant gene coincides with the genetic sex (i.e. male dominant gene and male sex) the personality traits are in greater harmony with the traits of the sex [2].

The Model also makes Type-specific nutritional recommendations, based on the brain function, the lack of certain micronutrients and the development of certain psychological disorders [2].

The objectives of the intervention were: to facilitate the children's adaptation in the kindergarten class by developing their self-awareness and hetero-awareness. The reduction of abusive phenomena, improvement of cooperation amongst children, as well as the improvement of certain children's, who were diagnosed with developmental disorders, ability to collaborate with their classmates were additional objectives. For this purpose, action research was considered as the most appropriate research methodology, since it constitutes an educational research process involving the teacher as researcher and aiming at bringing positive change both in themselves and their pupils [7].

\section{The Research}

The intervention took place as a health education program entitled "Getting to know myself and my classmates through the Paschalidis Tri-AnthropoType Model", from January since April 2014, two hourly twice a week. The class teacher was the main person responsible for the intervention and was assisted by G. D. Pashalidis as facilitator/ external collaborator, offering her regular feedback concerning her practice and research, and by a critical friend, who helped mainly in the reflection phases.

The intervention was connected to the following school subjects: language, mathematics, social sciences and creative expression.

\subsection{The identity of the class}

The kindergarten class consisted of 19 children (12 boys and 7 girls), aged 5 to 6, in Southern Attica, Greece. All children understood and spoke Greek. Eight of them, constituting a $42,5 \%$ of the class, either showed signs of certain disorders or had been officially diagnosed as suffering from them. Specifically: BOY I showed signs of hyperactivity and BOY II of attention deficit disorder and nervousness, but, to our knowledge, there had not been any official diagnosis for either of them. Six children had been officially diagnosed as having 
speech disorders and communication difficulties. Specifically: BOY 1 having articulation problems, dyslalia, anagrammatism and tachylalia, BOY 2 having logorrhoea, anagrammatism and difficulties in verbal expression, BOY 3 having dyslalia and anagrammatism, BOY 4 having dyslalia, GIRL 1 having dyslalia, sigmatism and rhotacism and GIRL 2 having dyslalia and sigmatism. The rest of the children of the class did not appear to have any particular behavioural problems or developmental disorders.

\subsection{Methodology, materials, activities}

Observations, questionnaires, structured interviews with the children and their parents/guardians, video recordings, the class teacher's journal and the children's portfolios were the research tools used. The teacher registered every child's initial attitude and progress and commented on it. She also took pictures of problematic situations, which were used as stimuli for discussion and collaborative solution seeking. She discussed the data with G.D. Paschalidis and with the critical friend. The portfolios provided material for the parents/guardians - teachers' discussions during their monthly meetings.

Triangulation was used to ensure intersubjectivity of the findings. In particular, three different ways of data collection were used (observation, questionnaire, structured interview) and data was collected from three different sources (teacher, children and their parents/guardians).

The research process consisted of two main steps. First step: The class teacher attended an introductory training course on the application of the Paschalidis Tri-Anthropo-Type Model in preschool education, taught by G.D. Paschalidis himself, where she was provided with the necessary guidelines and material for the intervention. The material included detailed information about every personality Types' traits and behaviour during the educational process. The teacher could identify every child's personality Type by examining many aspects of their school daily life, such as opening circle time, free play in designated areas, activities in the school yard, working on various projects, presenting group work to the rest of the class, reading time, leaving school.

Second step: The class teacher introduced the children to the Model through three sections of activities entitled: "Getting to know the Paschalidis Tri-Anthropo-Type Model", "Getting to know my personality Type", "Getting to know the personality Types of my schoolmates and my teacher and applying the Model in my class". In the first section of activities, the series of the tale - type stories by G.
D. Paschalidis were mainly used. The animal characters of the stories, representing the three distinct personality Types, helped the children identify them. Two research tools were mainly used in the second section of activities: structured interviews with the children and their parents/guardians and The Paschalidis Tri-AnthropoType Questionnaire for Preschool Children. A great variety of activities were included in the third section. After having identified their own personality Type, children learned the personality Type of their teacher and classmates. They counted how many of their classmates belonged to each personality Type, created indexes containing children's names and personality Types, etc. The series of the tale - type stories were used as inspiration for artwork, such as drawings, mask and puppet making, and dramatization followed by presentations in the class and in the neighboring kindergarten. The "Treasure of the Types" was created, a box containing children's artwork, expressing all their positive comments, thoughts and feelings concerning the personality Types. Two stories were created collectively by the class, entitled "The three personality Types go to kindergarten" and "Jungle Animals and Personality Types". Activities aiming at introducing the children to the nutritional principles of the Model and the Type-specific nutritional requirements followed.

Teamwork and cooperative learning constitute fundamental principles of the Model, which suggests that groups should consist of children of all three personality Types. The class teacher experimented on this principle by forming single-Type groups and mixed-Type groups and giving them a specific task to complete (to give a title to certain paintings).

\section{Results}

The structured interviews as well as the questionnaires used, showed that $15,79 \%$ of the children belonged to Type A, 52,63\% to Type B and $31,58 \%$ to Type C. The class teacher was identified as belonging to Type $\mathrm{B}$.

The class teacher observed and documented in her class journal positive change in the children's behavior, as a result of the intervention. By knowing the personality Type they belonged to, as well as the personality Types of their classmates and of their teacher, children became more aware of themselves and of the other. The teacher writes in her diary: "When they realized the existence of Type-specific strengths and weaknesses (extreme behaviors) they were amazed. As a result of this knowledge, every time a child showed a "non- accepted" Type-specific behavior (i.e. stubbornness, for Type $\mathrm{C}$ children or 
hyperactivity for Type A) the others recognized it as such and did not mimic it, as they used to do before the intervention. On the contrary, they commented on it as a Type-specific weakness, thus helping the misbehaving child to correct it. There was a radical change in the relationships in the class, which were gradually turning into relationships characterized by mutual understanding, friendship and collaboration."

As far as the teacher's initial difficulties with the class are concerned, the intervention provided evidence of the situation changing significantly to the better. Specifically: Type A children, who most of the time used to be rather hyperactive and unwilling to cooperate, started to behave more calmly and to collaborate more willingly with their classmates. Type B children, who most of the time used to be reserved and shy, showed more selfconfidence and, in the words of their teacher: "They raised their eyes up, expressed their opinion more loudly, so that it could be heard, and, at times, they even demanded their classmates to follow their suggestions for play." Type C children, who most of the time used to show signs of stubbornness, improved in that respect, collaborated in a more relaxed manner.

As far as the class atmosphere is concerned, the teacher notes: "The atmosphere of the class was transformed. An environment of acceptance and collaboration was soon established and it manifested itself throughout the intervention and beyond, till the end of school year. Despite the prevailing egocentricism of the children of that age, they stopped abusing each other and managed to collaborate and share." The overall effect was that children could now be creative and make the best use of their time in the kindergarten.

As far as teamwork and cooperation amongst children is concerned, both the teacher and the children found that the single-Type groups did not manage to complete the given task. In the "Type A" group there was tension and irritability. In the "Type B" groups there was too much talking and indecisiveness, whereas in the "Type C" groups there was competitiveness and stubbornness. When mixedType groups were formed, the children collaborated easily, worked enthusiastically and completed the given task in time.

As far as the children with certain disorders are concerned, our research showed: BOY I was identified to belong to Type A. During the intervention he gradually started behaving more calmly and taking part in collaborative activities. BOY II belonged to Type B and became more attentive and gradually less nervous. BOY 1's condition (Type A) remained unchanged. BOY 2 (Type B), started being less nervous when speaking, so that he could be more easily understood by his classmates, who stopped asking him to repeat his words. BOY 3 (Type B) improved his self-esteem and self-confidence according to his parents, his classmates and his teacher. BOY 4 (Type C) became more sociable and showed less signs of abusive behaviour. GIRL 1 (Type B) started showing less signs of embarrassment when speaking, despite the fact that her speech problems remained unchanged. GIRL 2 (Type B) improved her class participation and cooperation skills, despite her difficulties.

As far as the children's self-awareness and hetero-awareness are concerned, the teacher notes: "After realizing the characteristics of the three personality Types through working with the series of the tale - type stories, the children started to observe their own (self-observation) and their classmates' (hetero-observation) reactions, words and preferences and relate them to the personality Types. For example, when studying jobs, occupations and professions, it was noted that the children expressed their preference to those attributed by the Model to their personality Type. They could now identify similarities with those of their classmates who belonged to the same personality Type, as well as differences with those who belonged to the other two Types. Children were fascinated when they realized that Type A children's quick-temper, Type B children's sulking and Type C children's insisting were in fact Type-specific weaknesses." Role-play was also used, to help children explore the personality traits of the three Types, their strengths and weaknesses. Children used it as an opportunity to offer ideas and suggestions for changing Typespecific problematic behaviors.

As far as the parents' involvement is concerned, the teacher notes: "Most parents said that their children came back home happier, talked to them about the personality Types and expressed a wish to know or guess which personality Type their friends and other people in their environment belonged to. An $88 \%$ of the parents/guardians expressed an interest to learn more and, as they said, they would happily attend Tri-Anthropo-Type Paschalidis Model seminars for parents/guardians."

The class teacher also comments on an unexpected effect of the intervention on the neighboring school. "We were in the third week of the intervention when colleagues of the neighboring Kindergarten, which we share the school yard with, told me that they had noticed a significant change in my class children's behavior in the school yard. It was obvious for them that the children's play was less aggressive and that they could resolve their conflicts easier and quicker than before." 
The effect of the intervention on the class teacher as a professional was also significant. She comments in her journal: "I now feel more relaxed, since the children interact between themselves and with me in a more peaceful manner. I am happier and I enjoy my job. It is great to know who you are; it's the beginning of a creative and truthful existence." She also reported that her collaboration with the parents who were aware of the Model was very efficient, to the benefit of the children.

\section{Discussion-Limitations}

Our findings show that the intervention helped every child in the class to look at themselves and the other from a broader perspective. They also show that most of the children, by identifying strengths and weaknesses and attributing them not to themselves entirely but mainly to their personality Type, tried consciously to rely on their strengths and minimize their weaknesses. This process increased their self-awareness and self-respect, helped them cultivate their individuality as personalities and understand the other. We also found that mimicry of inappropriate behavior was drastically reduced and the class atmosphere was transformed into a happier one, in which friendship and collaboration were now obvious. Acceptance and understanding of the other was promoted.

The class teacher's work was facilitated, in that she could now work more efficiently and devote more time to constructive learning activities rather than to managing class conflict and problem solving. Her professional stress was reduced and she got pleasure from her work.

The positive impact of the application of the Paschalidis Model in preschool education in the specific kindergarten, constitutes strong evidence that application in any similar environment can have similar effect.

However, it would be interesting to elicit quantitative as well as qualitative data, which should be collected and analyzed with the appropriate research tools. Further research would also benefit from the teacher's collaboration with an academic institution (i.e. University).

This action research study constituted an experience of positive change for both the teacherresearcher and the children. Therefore, we feel we can recommend a generalized application of the TriAnthropo-Type Paschalidis Model, which would improve the learning environment in class and help teachers work more efficiently and happily and children develop their social skills, personality, understanding of themselves and the other.

\section{References}

[1] A. Stathopoulou, and G. Paschalidis, "The model of the three human personality types: a unique tool for the understanding of the individual differences in the teachinglearning process", Proceedings of 5th International Conference of Education, Research and Innovation, Madrid, 2012, pp. 2050-2059

[2] A. Stathopoulou, and G. Paschalidis, "Genetically determined differences of the three structures of limbic brain system determine three distinctive and unique personality types: a special key for understanding connection between brain function and its plasticity with health prevention", Proceedings of 18th International Neuroscience and Biological Psychiatry Conference, New Orlean, 2012, pp. 22-23

[3] G. Paschalidis, "Teaching Diverse Minds Successfully: The Paschalidis Tri-Anthropo-Types Model in Education (PTATM-E)", Proceedings of the World Congress on Education, 2013

[4] M. Shenton et al., "A Review of MRI findings in schizophrenia", Schizophrenia Research, Elsevier Science Ltd, Amsterdam, 2001, 49 (1-2), pp. 1-52

[5] S. Qin et al., "Amygdala Subregional Structure and Intrinsic Fuctional Connectivity Predicts Individual Differences in Anxiety During Early Childhood", Biological Psychiatry, 75 (11): 892

[6] M. Atmaca et al., "Neurochemistry of the hippocampus in patients with obsessive-compulsive disorder", Psychiatry and Clinical Neurosciences, Japanese Society of Psychiatry and Neurology, Tokyo, 2009, 63: pp 486-490

[7] Cohen, L., Manion, L., and Morrison, K, Research Methods in Education, Routledge, Oxon, 2011 